\title{
INFLUÊNCIA DA CORRELAÇÃO TEMPORAL E DA MULTIPLICIDADE DE TESTES NA DETECÇÃO DE TENDÊNCIAS DE ÍNDICES DE CHUVA NO TERRITÓRIO BRASILEIRO
}

\author{
SOUZA, Saulo Aires de - sauloaires@gmail.com \\ Universidade de Brasília/ UnB \\ AMORIM, Renato Souza - renatosamorim@gmail.com \\ Departamento Nacional de Infraestrutura de Transportes/ DNIT \\ REIS, Dirceu Silveira - dirceu.reis@gmail.com \\ Universidade de Brasília/ UnB
}

\begin{abstract}
RESUMO: A mudança no padrão das variáveis hidrometeorológicas, em particular de seus extremos, pode causar impactos em diversos setores da sociedade que são sensíveis às condições de tempo e clima, tais como o de recursos hídricos, agricultura, energia, transporte e saúde. Um dos mecanismos para compreender tais mudanças consiste em realizar estudos de detecção de tendências em séries temporais de variáveis hidrometeorológicas. Este artigo tem como objetivo demonstrar a influência da correlação temporal e da multiplicidade de testes de hipóteses nos resultados obtidos nesses estudos. A influência desses dois fatores é avaliada num estudo de detecção de tendência de dez índices de precipitação, empregando dados observados em 2481 estações de monitoramento espalhadas em todo o território brasileiro. Para lidar com a correlação temporal foram empregados os métodos Pre-Whitening e Trend-Free PreWhitening, enquanto que para a questão da multiplicidade, foi utilizado o controle baseado no False Discovery Rate (FDR). Os resultados mostraram que tanto a correlação temporal, quanto a questão da multiplicidade, se não forem consideradas na análise de detecção, podem distorcer de forma considerável o número de resultados identificados com presença de tendência, levando a uma interpretação equivocada da situação. Negligenciar esses fatores resulta sempre numa superestimativa do número de estações com tendência devido ao número elevado de falsas detecções, o que pode gerar decisões equivocadas na alocação de investimentos em estratégias de adaptação.
\end{abstract}

PALAVRAS-CHAVE: detecção de tendência; correlação temporal; análise regional; taxa de detecções falsas.

INFLUENCE OF TEMPORAL CORRELATION AND MULTIPLICITY OF TESTS IN TREND DETECTION OF PRECIPITATION INDICES IN BRAZILIAN TERRITORY

ABSTRACT: Daily precipitation recorded in 2481 gauges, with at least 30 years of record length and located throughout the Brazilian territory, were used to illustrate how the presence of temporal correlation and the method used to control the type 1 error, in situations where several hypotheses tests are carried out simultaneously in a region (multiplicity), affect the results of trend detection studies. Although widely reported in the literature, these factors are rarely considered in trend detection studies, especially in Brazil. The goal is to call the attention of the community to these factors. In this study, Pre-Whitening and Trend-Free Pre-Whitening are used to deal with temporal correlation, while the False Discovery Rate is employed to control the type 1 error in the regional analysis. This paper shows that if temporal correlation and the multiplicity problem are not considered in the analysis, the results of trend detection can be severely distorted resulting in erroneous interpretation of reality. Not considering these factors results in overestimation of the number of gauges with trends due to the large number of false detections, which may lead to equivocal investment allocation in adaptation strategies. 
KEYWORDS: Monotonic trends, temporal correlation, regional analysis, false discovery rate.

\section{INTRODUÇÃO}

A mudança no padrão das variáveis hidrometeorológicas, em particular de seus extremos, pode causar impactos em diversos setores da sociedade que são sensíveis às condições de tempo e clima, tais como o de recursos hídricos, agricultura, energia, transporte e saúde. A alteração na magnitude e frequência de veranicos numa dada região, por exemplo, pode causar prejuízos elevados no setor de agricultura de sequeiro, podendo inclusive inviabilizar a produção de determinada cultura naquele local. As mudanças em outras caraterísticas da precipitação podem resultar em alterações no regime de vazões mínimas em rios, refletidas no padrão estatístico de suas séries, resultando em uma diminuição da confiabilidade de sistemas de abastecimento de água e de irrigação, uma redução na capacidade de geração de energia hidrelétrica, e uma restrição no transporte de mercadorias via navegação fluvial.

Um dos mecanismos para compreender tais mudanças consiste em realizar estudos de detecção de tendências em séries temporais de variáveis hidrometeorológicas, ou de índices extraídos de tais séries. A detecção de tendências permite identificar se a variável de interesse tem experimentado uma mudança ao longo do período histórico. A identificação e estimativa de tal mudança pode servir de base para o desenvolvimento de estratégias de adaptação, como no caso de alteração na magnitude e frequência de chuvas intensas, quando é necessário avaliar possíveis medidas estruturais e nãoestruturais que possam reduzir os riscos de cheias.

Estudos de detecção de tendência são normalmente realizados com base em testes estatísticos aplicados à série temporal da variável de interesse (KUNDZEWICZ e ROBSON, 2004; KOUTSOYIANNIS, 2006). Tais testes são baseados na distribuição nula da estatística do teste, obtida a partir da premissa de que a hipótese nula $\left(H_{0}\right)$ é verdadeira, ou seja, de que a série não possui tendência. Com base na distribuição nula da estatística do teste e de sua estimativa amostral, decide-se se a tendência está presente (rejeição de $\mathrm{HO}$ ) ou não (aceitação de $\mathrm{HO}$ ) na série analisada. Não há certeza nessa decisão, pois é possível rejeitar $\mathrm{HO}$ quando não existe tendência alguma na série (erro tipo 1) ou aceitar erroneamente $\mathrm{HO}$ quando de fato a série contém uma tendência (erro tipo 2). Normalmente, o que se costuma fazer é controlar a probabilidade de se cometer o erro tipo 1 por meio da definição a priori do nível de significância do teste, ignorando por completo o erro tipo 2.

Há diversos desafios inerentes ao processo de detecção de tendências, e alguns desses desafios são frequentemente negligenciados em estudos dessa natureza realizados no Brasil. Dois desses desafios são abordados neste artigo, pois podem influenciar sobremaneira os resultados em estudos de detecção: (1) a presença de correlação temporal nas séries observadas, ou de índices baseados nas séries observadas, e (2) a questão conhecida na literatura estatística como multiplicidade em testes de hipóteses, associada ao controle do erro tipo 1 em situações onde são realizados diversos testes de hipóteses de forma simultânea. Esse último faz-se necessário levar em consideração, por exemplo, quando se deseja identificar o percentual de estações com tendência significativa numa dada região. 
Embora seja um problema conhecido de longa data (COX e STUART, 1955), não há uma solução definitiva para lidar com a correlação temporal em testes de hipóteses. Lettenmayer (1976) mostra que a presença da correlação temporal positiva resulta num aumento da probabilidade de se cometer um erro tipo 1, tornando-a maior do que o nível nominal de significância do teste, o que resulta num aumento de falsos positivos, conforme demonstrado em von Storch (1995) e constatado em estudos posteriores (DOUGLAS et al., 2000; YUE et al., 2002; BAYAZIT e ÖNOZ, 2007; KHALIQ et al., 2009; HAMED, 2009 e ONOZ e BAYAZIT, 2012). A literatura apresenta duas estratégias para lidar com esse problema.

A primeira estratégia consiste na modificação da série original por meio da remoção da autocorrelação, sendo o teste posteriormente aplicado à série modificada para avaliar a presença de tendência. O Pre-Whitening (PW), desenvolvido por Kulkarni e Von Storch (1995), é um exemplo dessa estratégia. Kulkarni e von Storch (1995) mostraram, via estudo de simulação Monte Carlo, que o uso do PW permite controlar adequadamente a probabilidade de se cometer o erro tipo 1. Porém, mais tarde, Yue e Wang (2002) concluem que a remoção da correlação temporal realizada no $\mathrm{PW}$ acaba por reduzir o poder do teste, que é a probabilidade de identificar a tendência (rejeitar HO) quando a tendência está de fato presente. Essa análise levou Yue et al. (2002) a sugerirem um outro método, denominado Trend-Free Pre-Whitening (TFPW), que resulta em maior poder de teste, quando comparada à PW, mas ainda assim com um adequado controle da probabilidade do erro tipo 1.

A segunda estratégia para lidar com a correlação temporal se baseia em alterações na distribuição nula da estatística do teste de hipóteses. Essa abordagem pode ser executada de diversas formas, como por exemplo, através da correção da variância da estatística de teste de Mann-Kendall, como realizado por Hamed e Rao (1998), com o emprego de uma nova distribuição, conforme derivado analiticamente por Hamed (2009), ou ainda por meio de um processo de reamostragem com reposição agrupada, bootstrap em blocos, como sugerido por Onoz e Bayazit (2012).

Apesar do elevado número de estudos de detecção de tendência de precipitação realizados no Brasil, poucos trabalhos trataram dessa questão (MULLER et al., 1998; MARENGO et al., 2008; TEIXEIRA e SATYAMURTY, 2011; BLAIN, 2013; BLAIN, 2014; ROSIN et al. 2015; PEDRON et al., 2017; CHAGAS e CHAFFE 2018). Os procedimentos utilizados envolveram, na maioria dos casos, a aplicação dos procedimentos PW e TFPW (BLAIN, 2014; ROSIN et al., 2015; CHAGAS e CHAFFE, 2018), com exceção de um procedimento Monte Carlo combinado com um modelo autoregressivo (MULLER et al., 1998).

O segundo fator que pode alterar fortemente os resultados em estudos de detecção de tendência é aquele chamado de multiplicidade em testes de hipóteses. Ignorar a questão da multiplicidade pode resultar num número excessivo de resultados falso positivos. Por exemplo, suponha a realização de um estudo de detecção de tendência numa região com 50 estações, todas elas sem a presença de tendência. O analista decide que o nível de significância do teste em cada estação deve ser de $5 \%$ e que a decisão de rejeitar ou aceitar $\mathrm{H}_{0}$ será baseada apenas no resultado do teste local, ignorando os resultados obtidos para as outras estações na região. Neste caso específico, se as séries em cada uma das estações forem independentes uma das outras, a 
probabilidade de se obter pelo menos um resultado falso positivo segue uma distribuição binomial (LIVEZEY;CHEN, 1983), e essa probabilidade é igual a $92 \%$, um valor bastante elevado, o que pode levar a interpretações equivocadas sobre o que acontece numa dada região.

A questão da multiplicidade é antiga na literatura estatística e muitas propostas foram desenvolvidas ao longo do tempo (HOLM, 1979; SIMES, 1986; HOCKBERG, 1988). Nos últimos anos, observou-se a popularização do método denominado false discovery rate (FDR), baseado no valor esperado da proporção do número de falso positivos dentre as hipóteses rejeitadas. O termo FDR passou a ser utilizado somente após Soriç (1989), e foi popularizado por Benjamini e Hochberg (1995). Largamente utilizada na genética e epidemiologia, a abordagem de controle de erros do tipo 1 por meio do FDR é relativamente nova nas ciências atmosféricas e na hidrologia, como nos trabalhos desenvolvidos por Alpert et al. (2002), Ventura et al. (2004), Wilks (2006), Renard et al. (2008), Fatichi (2009), Khaliq et al. (2009), Miao et al. (2012), Sun et al. (2014), Ganguly e Ganguli (2015), Gudmundsson e Seneviratne (2015), Cammalleri et al. (2016), Merz et al. (2016), Humphrey et al. (2016), Mallya et al. (2016), Wilks (2016) e Amorim (2018).

Tais trabalhos aplicam o conceito do FDR em seus estudos de detecção ou analisam a importância do método no controle do erro tipo 1 em estudos regionais de detecção de tendência. No Brasil, em praticamente todos os estudos que tenham avaliado a proporção de resultados significativos em determinada escala espacial, como forma de avaliar regionalmente eventuais mudanças nas séries, a questão da multiplicidade foi totalmente negligenciada, com exceção de apenas três estudos (CLARKE, 2004; BUARQUE et al., 2010, BOMBARDI e CARVALHO, 2017), o que reforça a necessidade de uma maior discussão acerca desse tema.

Este artigo tem como objetivo demonstrar a influência da correlação temporal e da multiplicidade de testes de hipóteses nos resultados obtidos em estudos de detecção de mudanças em variáveis hidrometeorológicas. A influência desses dois fatores é avaliada num estudo de detecção de tendência de dez índices de precipitação, empregando dados observados em 2481 estações de monitoramento espalhadas em todo o território brasileiro.

\section{MATERIAL E MÉTODOS}

\subsection{DADOS EMPREGADOS}

Neste artigo foram utilizados os registros históricos diários das estações pluviométricas disponíveis no banco de dados HIDROWEB da Agência Nacional de Águas (ANA). A seleção das estações baseou-se no seguinte critério: ter no mínimo 30 anos de dados sem falha ao longo do período histórico, e no mínimo, 5 anos de dados sem falha entre os anos de 2000 a 2015, resultando num total de 2481 estações. A Figura 1 apresenta a localização das estações empregadas neste estudo.

A maioria das estações selecionadas (56,5\%, 1402 estações) tem entre 30 e 44 anos de dados sem falhas (em vermelho). Dos 43,5\% das estações restantes (1079 estações), 21,8\% (540 estações) têm entre 45 e 59 anos (em amarelo), enquanto $21,7 \%$ possui mais de 60 anos (azul escuro). 
A análise espacializada dos resultados deu-se a partir das doze regiões hidrográficas brasileiras: Amazônica (AMZ), Parnaíba (PNB), Atlântico Nordeste Ocidental (ANC), Paraguai (PRG), Atlântico Sudeste (ASD), Tocantins-Araguaia (TOA), Atlântico Sul (ATS), Atlântico Nordeste Oriental (ANO), Uruguai (URU), São Francisco (SFR), Paraná (PRN) e Atlântico Leste (ALE). Tais regiões correspondem a bacias, grupo de bacias ou sub-bacias hidrográficas próximas, com características naturais, socais e econômicas similares (Resolução CNRH no 32 , de 15 de outubro de 2003). A motivação principal em utilizar como recorte espacial as regiões hidrográficas devem-se à importância que eventuais mudanças nas variáveis hidrometeorológicas têm na orientação do planejamento e gerenciamento dos recursos hídricos em todo o país.

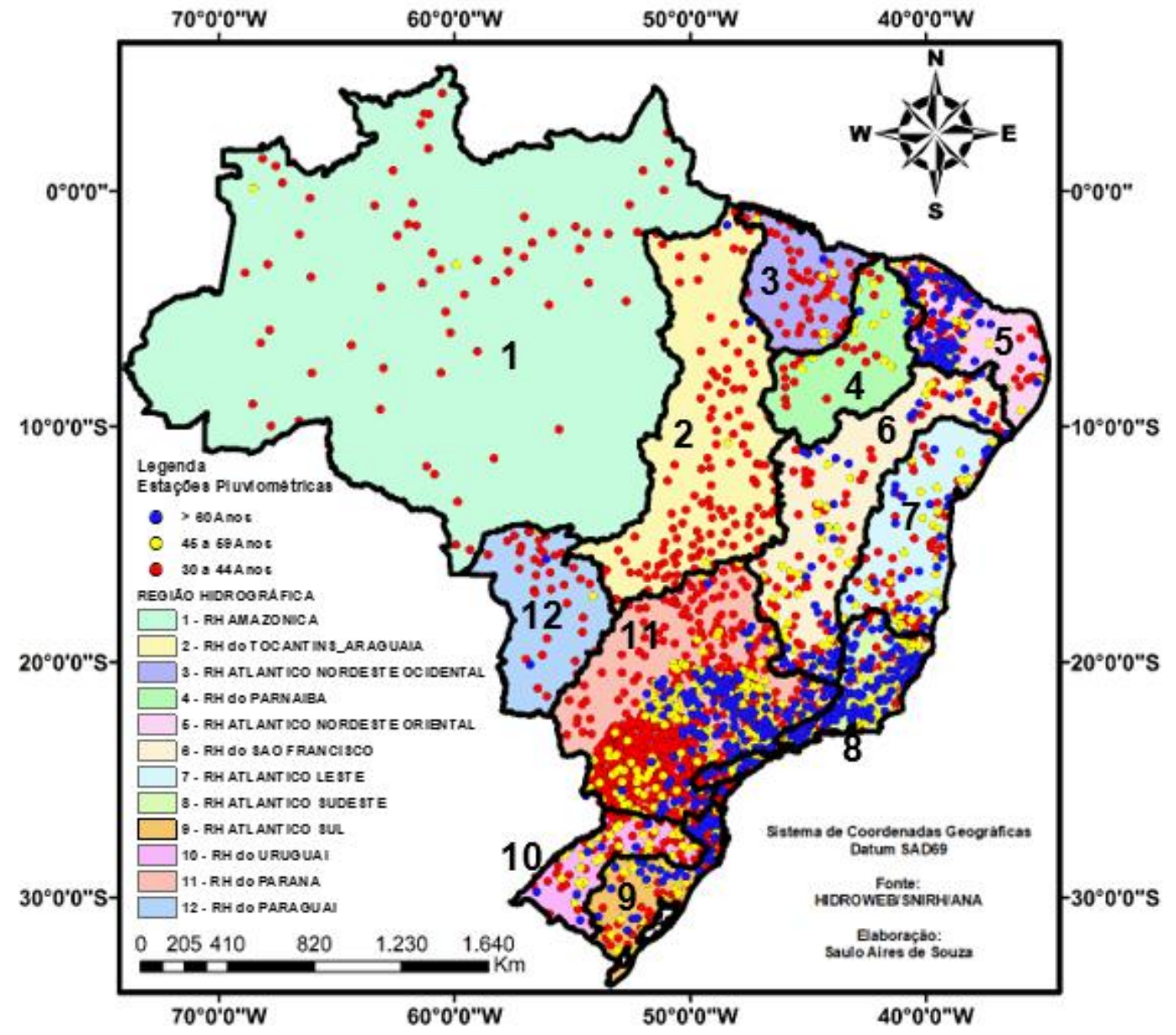

Figura 1 - Localização das estações pluviométrica utilizadas neste estudo.

\section{2 ÍNDICES DE PRECIPITAÇÃO}

A Tabela 1 apresenta uma descrição dos dez índices de precipitação empregados neste estudo. Esses índices foram selecionados a partir de uma lista maior com 27 índices baseados em valores diários de precipitação e temperatura (ZHANG et al., 2011, Tabela 1), preparada pelo grupo ETCCDI (Expert Team on Climate Change Detection and Indices) para servir de base para estudos de detecção de mudanças do clima, especialmente com foco em 
extremos. Com exceção do PRCPT, que pode ser considerado um índice padrão e imprescindível à avaliação pluviométrica em qualquer região, todos os outros índices procuram descrever algum aspecto de extremos de precipitação.

O CDD sinaliza vulnerabilidade às secas provocadas pelas longas estiagens, estimuladas pela evapotranspiração excessiva e perda precoce dos volumes hídricos nos reservatórios, demandando políticas públicas emergenciais. Esse índice está associado a prejuízos às atividades agropecuárias e industriais, e ao abastecimento doméstico, algumas vezes com racionamento do consumo, além do estímulo à recessão econômica provocada pela escassez dos insumos de produção. O CWD, por sua vez, é importante para a avaliação das interrupções das estiagens e a manutenção ecológica das áreas mais secas, por exemplo. Os índices R10, R20, R50 podem ser vistos como indicativos das ocorrências de eventos extremos de precipitação, provendo uma ideia de possibilidade de alagamentos, inundações e deslizamentos de encostas. 0 RX1day e RX5day são indicadores de situação de emergência ou calamidade pública, associados a eventos críticos de precipitação causadores de danos econômicos e sociais.

Tabela 1 - Índices de precipitação empregados no estudo.

\begin{tabular}{cc}
\hline Índice & Descrição \\
\hline PRCPT & Total de precipitação no ano. \\
SDII & PRCTOT/R1 \\
\hline R1 & Número de dias com precipitação igual ou superior a $1 \mathrm{~mm}$. \\
\hline R10 & Número de dias com precipitação igual ou superior a $10 \mathrm{~mm}$. \\
\hline R20 & Número de dias com precipitação igual ou superior a $20 \mathrm{~mm}$. \\
\hline R50 & Número de dias com precipitação igual ou superior a $50 \mathrm{~mm}$. \\
\hline CDD & Número máximo de dias consecutivos secos $(\mathrm{P}<1 \mathrm{~mm})$ no ano. \\
\hline CWD & Número máximo de dias consecutivos úmidos $(\mathrm{P} \geq 1 \mathrm{~mm})$ no \\
& ano. \\
\hline RX1d & Máxima precipitação diária no ano. \\
\hline RX5d & Máxima precipitação acumulada ao longo de 5 dias no ano. \\
\hline
\end{tabular}

Fonte: Zhang e Yang (2004).

\subsection{DETECÇÃO E MAGNITUDE DE TENDÊNCIA}

\subsubsection{TESTE DE MANN-KENDALL (MK)}

A análise de tendência foi realizada utilizando o teste não-paramétrico Mann-Kendall (MK), um dos mais utilizados para a detecção de tendências monotônicas em séries temporais de variáveis hidrometeorológicas. $O$ teste $M K$ é pouco sensível à presença de outliers e não exige a definição de uma distribuição de probabilidades para os dados.

$O$ teste MK assume, como hipótese nula, que a série $X=\left\{X_{1}, X_{2}, \ldots, X_{n}\right\}$ é formada por variáveis independentes e igualmente distribuídas, sem a presença de tendência. Se a hipótese nula for verdadeira, a ordem das observações seria apenas uma possibilidade dentre as $n$ ! equiprováveis que poderiam ter acontecido. Com base nesse fato, Mann (1945) e Kendall (1975) desenvolveram a estatística de teste S, conforme descrito na equação 1 , 


$$
S=\sum_{i=1}^{n-1} \sum_{j=i+1}^{n} \operatorname{sign}\left(x_{j}-x_{i}\right)
$$

em que

$$
\operatorname{sign}\left(x_{j}-x_{i}\right)=\left\{\begin{array}{c}
1, \text { se } x_{j}>x_{i} \\
0, \text { se } x_{j}=x_{i} \\
-1, \text { se } x_{j}<x_{i}
\end{array}\right.
$$

estatística de teste $S$ não depende dos valores observados de $\mathrm{X}$, mas sim de seu posto, o que significa que nenhuma suposição é feita a respeito da natureza da distribuição de probabilidade que gerou a amostra. Isso explica o porquê do teste MK não ser muito sensível a valores amostrais discrepantes e ser capaz de manter o poder de detecção, mesmo quando a distribuição populacional é bastante assimétrica. Quando a hipótese nula é verdadeira, Kendall (1975) mostrou que, para valores de $n>10$, a distribuição de $S$ pode ser aproximada por uma distribuição Normal, $\mathrm{S} \sim \mathrm{N}\left(0, \sigma^{2}\right)$, com média nula e variância dada por:

$$
\sigma^{2}=\frac{n(n-1)(2 n+5)-\sum_{j=1}^{m} t_{j} j(j-1)(2 j+5)}{18}
$$

em que tj é o t-ésimo empate na série e m é o número de grupos de empates. Como $S$ é normalmente distribuído, então $S / \sigma \sim N(0,1)$. Entretanto, dado a nãocontinuidade de S, uma correção deve ser empregada. Kendall (1975) sugere a adição ou subtração de uma unidade para a correção de continuidade, conforme mostrado abaixo:

$$
Z=\left\{\begin{array}{c}
\frac{s-1}{\sigma}, \text { se } S>0 \\
0, \text { se } S=0 \\
\frac{s+1}{\sigma}, \text { se } S<0
\end{array}\right.
$$

em que $Z$ é a variável aleatória da Normal padrão. Assim, para um teste bicaudal com o nível de significância igual a a\%, se $Z<Z_{\alpha / 2}$ ou $Z>Z_{1-\alpha / 2}$, a hipótese nula é rejeitada e assume-se que a tendência é estatisticamente significante para a estação de monitoramento avaliada. O nível de significância adotado no estudo foi de $5 \%$.

\subsection{CORRELAÇÃO TEMPORAL}

Uma das principais dificuldades inerente à aplicação dos testes estatísticos na detecção de mudanças ocorre quando as séries são correlacionadas serialmente. A presença de correlação temporal positiva (negativa) resulta em rejeição da hipótese com uma frequência maior (menor) do que a estabelecida pelo nível de significância do teste (VON STORCH, 1995). Isso se deve à relação existente entre a variância da estatística do teste com o grau de autocorrelação da série (YUE et al., 2002).

A eliminação do efeito da correlação serial na detecção de tendência é um problema complexo devido à mútua interação entre a correlação e a tendência. Para lidar com essa problemática, foram consideradas no estudo duas abordagens: (i) Pre-Whitening (PW), sugerida inicialmente por Kulkarni e Von Storch (1995) e (ii) Trend-Free-Pre-Whitening (TFPW), introduzida por Yue 
et al. (2002). Ambas as técnicas recorrem a uma modificação da série original por meio da remoção da estrutura de correlação.

\subsubsection{PRE-WHITENING (PW)}

O procedimento PW é muito simples de ser aplicado e prescinde, para a obtenção de bons resultados, de que o conjunto de dados da amostra avaliada, $X_{t}$, possa ser expresso adequadamente por um modelo autoregressivo lag 1 , $A R(1)$, cuja definição formal é dada por:

$$
X_{t}=\theta X_{t-1}+\epsilon_{t}
$$

Em que $\theta$ é o coeficiente do modelo $\operatorname{AR}(1)$ e $\epsilon_{t}$ são os erros, considerados independentes e normalmente distribuídos, $N\left(0, \sigma_{\epsilon}^{2}\right)$. A metodologia proposta por Kulkarni e Von Storch (1995) pode ser realizada através dos seguintes passos: (a) Estimativa da autocorrelação amostral $\left(\hat{r}_{1}\right)$ por $r_{1}=\frac{\sum_{i=1}^{n-1}\left(X_{i}-\bar{X}\right)\left(X_{i+1}-\bar{X}\right)}{\sum_{i=1}^{n}\left(X_{i}-\bar{X}\right)^{2}}$; (b) se $\hat{r}_{1}$ for estatisticamente diferente de zero, o teste de MK é aplicado para a série modificada $X_{t}^{\text {mod }}=X_{t}-\hat{r}_{1} X_{t-1}$ para $\mathrm{t}=2, \ldots, \mathrm{n}$. Caso contrário, o teste $\mathrm{MK}$ é aplicado à série original.

\subsubsection{TREND FREE PRE-WHITENING (TFPW)}

Dadas as limitações constatadas no procedimento PW, Yue et al. (2002) sugerem o uso do TFPW, após a realização de uma série de estudos de simulação Monte Carlo. As conclusões resultantes do trabalho citado mostraram que remover a autocorrelação, conforme definido pelo procedimento $\mathrm{PW}$, implica na redução da magnitude da tendência presente na série modificada, resultando numa substancial diminuição no poder de detecção do teste.

Com o intuito de mitigar o problema relatado, Yue et al. (2002) sugerem a remoção da tendência estimada da série originalmente observada antes da aplicação dos procedimentos previstos pelo PW. O TFPW prescinde da realização dos seguintes passos metodológicos: (a) Estimativa da magnitude da tendência da série amostral, $\hat{\beta}$, a partir da aplicação do método não-paramétrico proposto por Sen (1968); (b) Remoção da tendência da série original $\left(X_{t}\right)$ e obtenção de amostra modificada, a partir da aplicação da expressão: $X_{t}^{\text {mod }}=X_{t}-\hat{\beta} t$; (c) Estimativa da autocorrelação amostral $\left(\hat{r}_{1}\right)$ da série modificada, $X_{t}^{\text {mod }}$; (d) Se $\hat{r}_{1}$ não é estatisticamente diferente de zero, então o teste de MK é aplicado à série original. Caso contrário, o procedimento de $\mathrm{PW}$ é aplicado a $X_{t}^{\text {mod }}$ resultando na série $Y_{t}^{\text {mod }}=X_{t}^{\text {mod }}-\hat{r}_{1} X_{t-1}^{\text {mod }}$; (e) A tendência é novamente devolvida à série $Y_{t}=Y_{t}^{\text {mod }}+\hat{\beta} t ;$ (f) Aplicação do teste de MK à $Y_{t}$.

\subsection{MULTIPLICIDADE DE TESTES}

Estudos de detecção de tendência consistem em aplicar simultaneamente testes de hipóteses em diversas estações localizadas numa dada região. Níveis locais de significância, $a_{L}$, são sempre definidos a priori, refletindo as preferências do analista em relação à possibilidade de cometer um erro ao 
declarar uma estação não-estacionária, quando na verdade nenhuma tendência está presente nos dados (erro tipo 1). Se a hipótese nula for verdadeira, a probabilidade de se cometer um erro tipo 1 é igual $a a_{L}$, previamente definido.

Definir a região de rejeição para um teste local com nível de significância $\mathrm{a}_{\mathrm{L}}$ é uma tarefa trivial, porém quando se deseja controlar o erro a nível regional, a tarefa se torna mais complicada, pois não fica claro qual erro deve ser controlado. Os dois procedimentos adotados aqui são baseados em um dos dois erros descritos na sequência, quais sejam, o PCER (Per-Comparison Type 1 Error Rate) e o FDR (False Discovery Rate), embora outros pudessem ter sido empregados, como por exemplo, o FWER (Family-Wise Type 1 Error Rate) (VENTURA et al., 2004).

O procedimento baseado no PCER ignora por completo o problema da multiplicidade, já que a decisão de aceitar ou rejeitar a hipótese nula é feita com base apenas na informação contida na própria estação, sem levar em consideração as decisões tomadas nas outras estações da região. Como não há qualquer perspectiva do que acontece na região, os resultados acabam por violar o nível nominal de significância a nível regional, $a_{R}$.

Benjamini e Hochberg (1995) propuseram uma abordagem diferente para lidar com o problema da multiplicidade. Eles sugeriram controlar o FDR, definido como sendo o valor esperado da proporção de rejeições falsas,

$$
F D R= \begin{cases}E\left[\frac{N_{10}}{R}\right], & \text { if } R>0 \\ 0, & \text { if } R=0\end{cases}
$$

em que $\mathrm{N}_{10}$ é o número de estações em que a hipótese nula foi rejeitada erroneamente e $R$ é o número total de rejeições na região. Controlar o FDR possui algumas vantagens. Por exemplo, o poder do teste é maior quando se emprega o FDR, quando comparado com outros tipos de controle, sendo os resultados mais fáceis de interpretar uma vez que esses não dependem do número de estações na região. Por exemplo, obter 4 rejeições falsas em 10 rejeições é mais preocupante do que obter 4 rejeições falsas em 50 rejeições. Controlar o FDR permite levar esse fato em consideração, o que não ocorre quando se decide controlar outros erros. Uma outra vantagem em utilizar o FDR é que tal procedimento é pouco sensível à correlação espacial, de modo que pode ser aplicado independentemente da existência ou não da dependência espacial entre estações (VENTURA et al., 2004).

O procedimento proposto por Benjamini e Hochberg (1995) baseia-se no ordenamento em ordem crescente dos $p$-valores obtidos quando da realização dos testes de hipóteses em cada uma das $m$ estações localizadas numa dada região, $P_{(1)} \leq P_{(2)} \leq \cdots \leq P_{(m)}$. Esses $p$-valores estão associados às hipóteses $H_{(1)} \leq H_{(2)} \leq \cdots \leq H_{(m)}$ e a um dado valor crítico $d_{1} \leq d_{2} \leq \cdots \leq d_{m}$, considerado igual a $d_{i}=i \alpha_{R} / m$. O procedimento é sequencial, o que significa dizer que a decisão de aceitar ou rejeitar a hipótese nula é tomada uma estação de cada vez, começando por aquela que possui menos evidência da presença de tendência, ou seja, com a que possui o maior $p$-valor. Se por acaso, $P_{(m)}<d_{m}=$ $a_{R}$, então todas as estações na região são consideradas não-estacionárias. Caso contrário, a condição $P_{(j)}<d_{j}$ para todo $(\mathrm{j}<\mathrm{m})$ é verificada até que a condição seja satisfeita. Quando isso acontece, todas as hipóteses $\mathrm{H}(\mathrm{i})<\mathrm{j}$ são rejeitadas. 


\section{RESULTADOS E DISCUSSÃO}

A fim de ilustrar a influência da correlação temporal e da multiplicidade de testes em análise regional de detecção de tendências, aplicou-se o teste nãoparamétrico de MK nas séries históricas dos 10 índices de chuva. Para isso, foram empregadas seis estratégias distintas: MK, MK-PW, MK-TFPW, MK-FDR, FDR-MK -PW, FDR-MK -TFPW. A estratégia denominada MK desconsidera por completo tanto a questão da autocorrelação quanto da multiplicidade dos testes. Essa estratégia, que é a mais frequentemente empregada em estudos de tendência realizados no Brasil, tende a obter um número mais elevado de deteç̧ões de tendências significativas. As estratégias MK-PW e MK-TFPW tratam apenas da questão da autocorrelação, ignorando a questão da multiplicidade de testes na análise regional. Por fim, as estratégias que envolvem o FDR incorporam a questão da multiplicidade. Neste estudo, tanto o nível de significância local, quanto o regional, foram estabelecidos em $5 \%$.

A Figura 2 apresenta, para cada um dos índices de precipitação avaliados, o percentual de estações que apresentaram tendência significativa, seja ela crescente ou decrescente. Para cada índice, são apresentados os resultados de cada uma das seis estratégias, permitindo avaliar o grau de influência da correlação temporal e da multiplicidade de testes na análise.

Os resultados mostram que desconsiderar a questão da correlação temporal e da multiplicidade de testes pode distorcer os resultados do teste de tendência. Para todos os índices, e para todas as estratégias que de alguma forma lidam com uma ou ambas as questões identificadas acima, observa-se uma redução na quantidade de resultados significativos quando comparado à estratégia MK. Essa redução, como era de se esperar, depende da estratégia empregada. Por exemplo, no caso do índice R1, que apresentou o maior percentual de estações significativas, quando se aplica a estratégia MK pura e simplesmente, conclui-se que 36,7\% (911 estações) das 2481 estações investigadas possuem tendência monotônica, um número bastante elevado. Entretanto, quando se considera a questão da correlação temporal e da multiplicidade, o percentual de estações identificadas com tendência diminui, e o grau de redução, depende da estratégia empregada.

A estratégia FDR-MK-TFPW, que lida conjuntamente com as questões da correlação temporal e multiplicidade de testes, quando empregada para analisar o índice R1, resulta na identificação de 23\% (575 estações) das estações com tendência significativa, uma redução relativa da ordem de $37 \%$, quando comparado com a estratégia MK (911 estações). Entretanto, essas reduções, quando se compara os métodos MK e FDR-MK-TFPW, são ainda maiores para outros índices, como por exemplo: PRCPT, que passa de 22,1\% (549 estações) para 7,3\% (172 estações), uma redução relativa de $65 \%$; R10, que passa de 22,1\% (549 estações) para 8,4\% (208 estações), uma redução relativa de $62 \%$; Rx1d, que passa de 15,3\% (379 estações) para 2,7\% (68 estações), uma redução relativa de $82 \%$. 


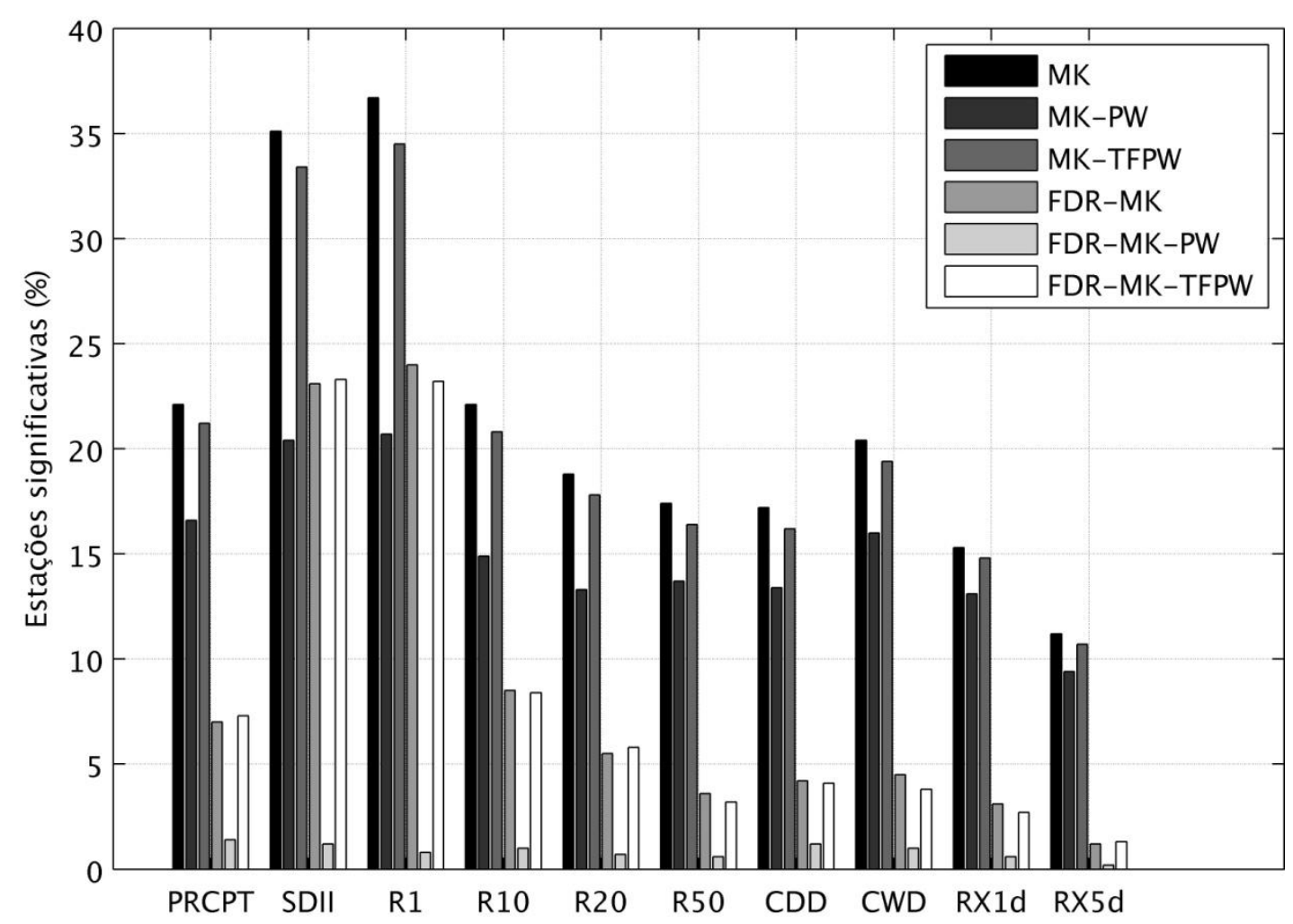

Figura 2 - Percentual de estações com tendência significativa (crescente ou decrescente).

Como era de se esperar, o uso do procedimento FDR, construído exatamente para restringir o valor esperado do número de resultados falso positivos em relação ao número de estações com resultados significativos ( $\mathrm{aR}=$ 0,05), resultou numa redução expressiva do número de estações identificadas com tendência. Os resultados mostram também que a questão da multiplicidade de testes teve um impacto maior no percentual de detecção de tendência do que a questão da correlação temporal. Para evidenciar essa questão, é preciso analisar primeiro os resultados obtidos com as estratégias que lidam única e exclusivamente com a correlação temporal, quais sejam MK-PW e MK-TFPW. Quando comparamos os resultados obtidos pelas duas estratégias, percebemos que o uso de PW para lidar com a correlação temporal resulta sempre num percentual menor de detecção de estações significativas.

A aplicação das estratégias MK-PW e MK-TFPW, como feito aqui, não permite identificar qual delas é a mais adequada, mas fica o registro de que os resultados obtidos podem ser bastante distintos, trazendo incertezas neste tipo de análise. Entretanto, vale mencionar que essa questão foi estudada por Yue et al. (2002) por meio de simulação Monte Carlo, tendo sido mostrado que o procedimento MK-PW, além de remover a autocorrelação, remove também uma parte da tendência, alterando a magnitude do $p$-valor, com repercussões também na identificação de estações significativas quando se aplica o FDR.

Independente do fato de ser utilizar PW ou TFPW para lidar com a questão da correlação temporal, pode-se notar que quando se emprega a 
estratégia FDR-MK, que negligencia a questão da correlação por completo, focando apenas na questão da multiplicidade, os percentuais de estações identificadas como significativas são bem inferiores daqueles obtidos pelas estratégias MK-PW e MK-TFPW, com exceção dos índices R1 e SDII. Por exemplo, enquanto o uso da estratégia MK-PW resulta na identificação de 16,6\% (397 estações) de estações significativas para o PRCPT, o uso do FDR-MK resulta em apenas 7,0\% (174 estações), uma diferença expressiva. E essas diferenças se repetem para outros índices, tais como: R10, que passa de $14,9 \%$ (MK-PW) para 8,5\% (FDR-MK); R50, que passa de 13,7\% (MK-PW) para 3,6\% (FDR-MK); e Rx1d, que passa de 13,1\% (MK-PW) para 3,1\% (FDR-MK). Esses resultados mostram de fato que a questão da multiplicidade se sobressai sobre a questão da correlação temporal, embora nos casos de R1 e SDII, isso não seja tão claro se adotarmos a estratégia MK-PW. Por exemplo, para o R1, o uso de MK-PW resulta em $20,7 \%$ de estações significativas, enquanto o uso de FDR-MK resulta em 24\%. Essa preponderância da questão da multiplicidade já era esperada, tendo em vista que os índices de precipitação costumam apresentar baixa persistência de curto prazo ou dependência temporal, diferentemente de algumas variáveis hidrológicas como as vazões médias e mínimas. Entretanto, a escolha da técnica a ser empregada para lidar com a questão da correlação temporal, PW ou TFPW, se mostrou importante.

Até aqui, a análise se ateve às diferenças de resultados em termos de percentual de estações com tendências significativas, sem qualquer discussão acerca da natureza das tendências, crescente ou decrescente, ou sobre a distribuição espacial das mesmas. A Figura 3 apresenta, separadamente, o percentual de estações significativas com tendências crescentes e decrescentes para cada um dos índices empregados, e apenas para as estratégias MK e FDRMK-TFPW. Como discutido anteriormente, independente se a tendência é crescente ou decrescente, quando se considera a correlação temporal e a multiplicidade de testes (FDR-MK-TFPW), o número de estações identificadas como significativas são significativamente menores do que quando se negligencia esses fatores (MK). 


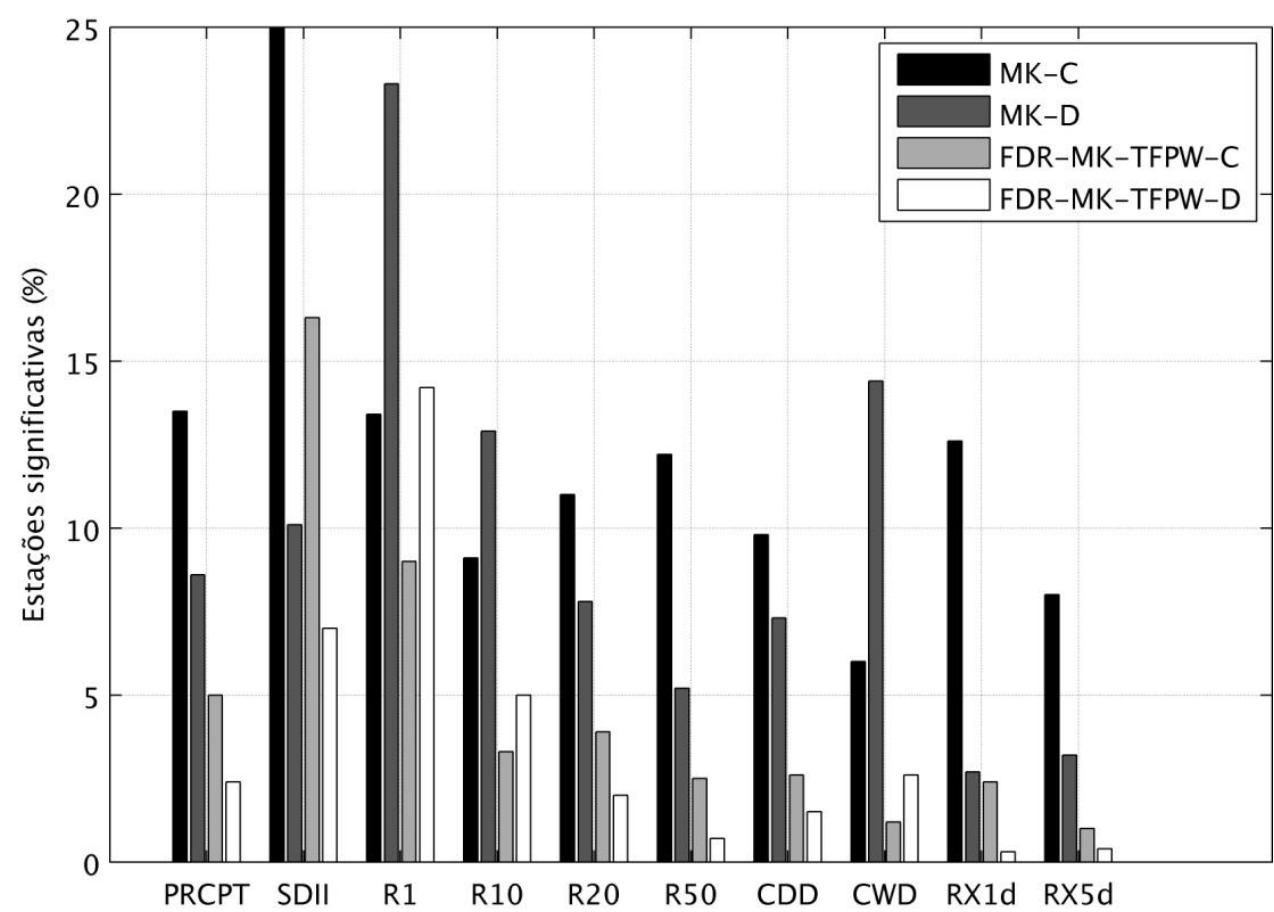

Figura 3 - Percentual de resultados significativos, separados por tendências crescentes e decrescentes, para as estratégias MK e FDR-MK-TFPW.

Por limitação de espaço, e por entender que a inclusão de uma análise regional detalhada de todos os índices não seria necessária para a compreensão do grau de influência da autocorrelação e da multiplicidade em estudos de detecção de tendência, as Figuras 4 e 5 apresentam apenas a distribuição espacial das tendências para os índices PRCPT e RX1d, considerando as seis estratégias empregadas. O primeiro índice (PRCPT) é muito empregado quando se deseja avaliar possíveis mudanças na disponibilidade de água na região, enquanto o segundo índice $(\mathrm{RX} 1 \mathrm{~d})$ é usualmente utilizado para avaliar a ocorrência e magnitude de cheias. Nessas figuras, os círculos vermelhos ilustram as estações com tendência decrescente significativa, os azuis as estações com tendência crescente significativa e os pretos as estações em que não foi possível rejeitar a hipótese nula.

Fica evidente, no caso do PRCPT, uma incidência de tendências crescentes mais ao sul do país, e de tendências decrescentes mais ao norte, com uma região de transição próxima à região sudeste. Já para o RX1d, observa-se uma preponderância maior de tendências crescentes espalhados em todo território nacional. Como já discutido anteriormente, nota-se uma redução importante quando se aplica o FDR em ambos os índices, embora o padrão de tendências crescentes mais ao sul e decrescentes mais ao norte, no caso do PRCPT, e o padrão crescente para o RX1d se mantêm. Observa-se também uma maior convergência regional nos resultados ao se utilizar o FDR, reduzindo o número de situações incongruentes, como estações vizinhas com resultados significativos de sinal contrário. Embora não tenha sido mostrado, por limitação de espaço, esse padrão espacial dos resultados relativo à aplicação das diferentes estratégias foi similar para os outros índices. A estratégia que se mostrou mais conservadora em termos de rejeição da hipótese nula considerando o recorte espacial por $\mathrm{RH}$, foi a que combinou a questão da 
autocorrelação e multiplicidade simultaneamente a partir da estratégia FDR-PW, independentemente do índice. Nessa estratégia, praticamente todas as $\mathrm{RH}^{\prime} \mathrm{s}$ tiveram mais de $90 \%$ de redução de resultados significativos em relação à estratégia MK, com exceção da RH ATS. Com relação a climatologia, observa-se que uma parte importante do padrão dessas tendências reportadas são na realidade resultados de flutuações naturais de curta ou longa escala temporal, tendo em vista que a consideração da autocorrelação e multiplicidade revelou um excesso na detecção dessas tendências.

De um modo geral, esses resultados concordam com a maior parte dos estudos de tendência realizados no Brasil no que se refere à distribuição espacial do sentido das tendências. Entretanto, quando se avalia a proporção de resultados significativos apresentados nesses estudos, observam-se valores mais elevados do que os apresentados aqui, como relatado na sequência.

Na escala nacional, os resultados obtidos por Haylock et al. (2006), Skansi et a.I (2013), Salviano et al. (2016), Penereiro e Meschiatti (2017) e Penereiro (2018), embora todos com um número bem menor de estações, quando comparados com o presente estudo, concordam com esse padrão geral de aumento das condições mais úmidas em boa parte das regiões hidrográficas localizadas no Sul/Sudeste, e de condições mais secas no Nordeste. Com relação ao percentual de rejeições, Haylock et al. (2006) detectaram cerca de $31 \%$ e 6\% para os índices PRCPT e RX1d respectivamente, números superiores aos apresentados no presente estudo, $7 \%$ e $3 \%$ para os mesmos índices, quando consideradas as questões de autocorrelação e multiplicidade. Salviano et al. (2016) reportaram também um percentual de 30\% para o PRCPT. Os outros estudos realizados a nível nacional, e mencionados acima, apresentaram também um percentual de rejeições levemente superior ao apresentado aqui, no entanto, além de diferenças no período de dados empregado, como em Skansi et al (2013), os estudos de Penereiro e Meschiatti (2017) e Penereiro (2018) foram realizados numa escala sazonal, diferente do que foi realizado no presente trabalho, o que torna a comparação com o estudo atual mais difícil.

Numa escala mais regional, como na região amazônica, Satyamurty et al. (2010), Alves (2016) e Espinoza et al. (2018) também relatam um padrão de tendências negativas ao sul e positivas ao norte, tanto no total de chuvas, quanto nos extremos, embora menos pronunciado do que o identificado aqui. A diminuição mais pronunciada dos totais precipitados nas regiões hidrográficas localizadas no Nordeste foi também reportada em Ferreira et al. (2017) para Pernambuco, e em Ferreira et al. (2018) e Hiera et al. (2019) para o Ceará, com percentuais de rejeição entre 16 a 30\%, bem superiores aos obtidos no presente estudo para essa região, que foi da ordem de $6 \%$. O aumento das condições mais úmidas e extremas da precipitação foi amplamente relatada nos estudos de Muller et al. (1998), Pinheiro et al. (2013), Zandonadi et al. (2014), Ávila et al. (2016), Chagas e Chaffe (2018) e Ely (2019). Em Chagas e Chaffe (2018) embora não explicitado no estudo, observou-se que os resultados tanto para o PRCPT como o RX1d concordaram em termos de rejeição com os resultados aqui apresentados, sendo que parte dessa concordância se deve tanto ao número de estações similares utilizadas, como também ao tratamento da questão da autocorrelação realizada.

Embora não tenha sido objeto de investigação do estudo, a atribuição das tendências identificadas, notadamente aquelas cujo o padrão regional é 
mais claro, como o aumento das condições mais úmidas no sul/sudeste/centrooeste e de condições mais secas no nordeste, vem sendo associadas à mudanças no padrão de variabilidade de fenômenos atmosféricos de larga escala decorrentes de interações oceano-atmosfera que modulam tanto na escala temporal como na escala espacial a precipitação (HAYLOCK et al., 2006; MARENGO E VALVERDE, 2007; ALVES et al., 2013; SKANSI et al., 2013; SALVIANO et al. 2016). Diversos estudos mostram que as condições das temperaturas da superfície do mar (TSM) sobre os oceanos tropicais influenciam o regime de chuvas do Nordeste, do Sul/Sudeste do Brasil e da Amazônia e que mudanças na frequência e intensidade desses eventos estão diretamente relacionados à essas mudanças observadas nos diferentes índices de precipitação utilizadas nesse estudo (HAYLOCK et al., 2006; MARENGO E VALVERDE, 2007; VALVERDE e MARENGO, 2014; SALVIANO et al. 2016; SALGUEIRO et al., 2016; PENEREIRO E MESCHIATTI, 2017). Por exemplo, o aumento das condições mais úmidas e extremas da precipitação tanto no Sul com em parte do Sudeste e Centro-Oeste no Brasil foram associados à mudanças de intensidade de eventos El Niño Oscilação Sul (ENOS) que por sua vez estão relacionados à modulação deste fenômeno com a oscilação decadal do pacífico (BARROS et al., 2008; CAVALCANTI E AMBRIZZI, 2009; ÁVILA et al., 2016), já no Nordeste a diminuição da precipitação também vem sendo associada a mudanças nos padrões de ocorrência da ENOS e também de alterações no padrão de anomalias da TSM sobre o oceano atlântico tropical, comumente chamado de padrão de dipolo do atlântico (HAYLOCK et al., 2006; SANTOS e BRITO, 2007; KAYANO e CAPISTRANO, 2013; SALGUEIRO et al., 2016). É importante destacar que as mudanças climáticas globais antropogênicas vêm sendo apontado como o principal indutor dessas mudanças nos padrões de variabilidade desses indicadores climáticos (IPCC, 2013; MARENGO et al. 2018).

Os resultados apresentados aqui demonstram alguns desafios na aplicação e interpretação dos resultados dos testes de tendência aplicados às séries hidrometeorológicas. É fundamental que essas incertezas sejam apresentadas no sentido de subsidiar o tomador de decisão na incorporação de tais incertezas para fins de definição de estratégias de adaptação no âmbito do planejamento de recursos hídricos. 


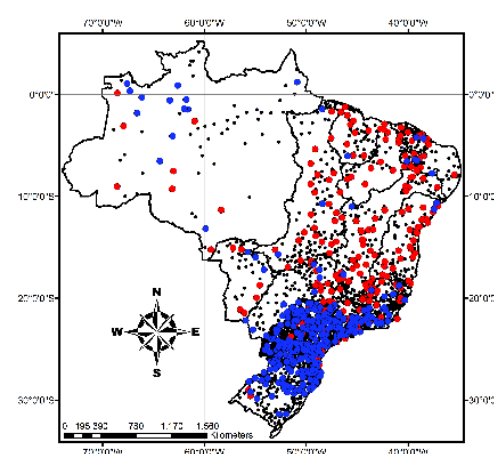

(a) MK

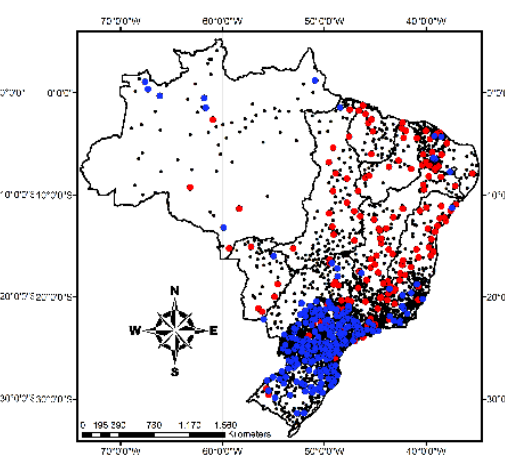

(b) MK-PW

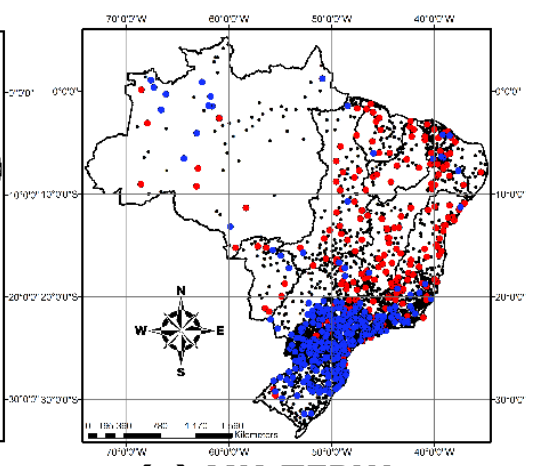

(c) MK-TFPW

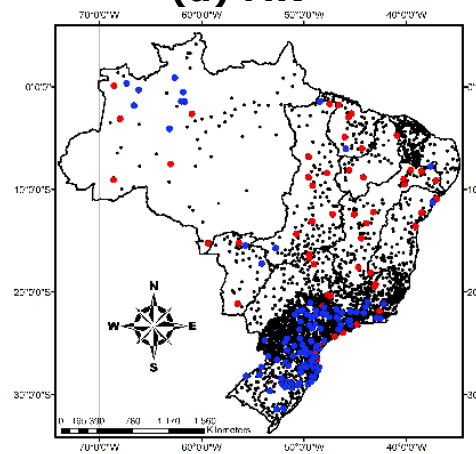

(d) FDR-MK

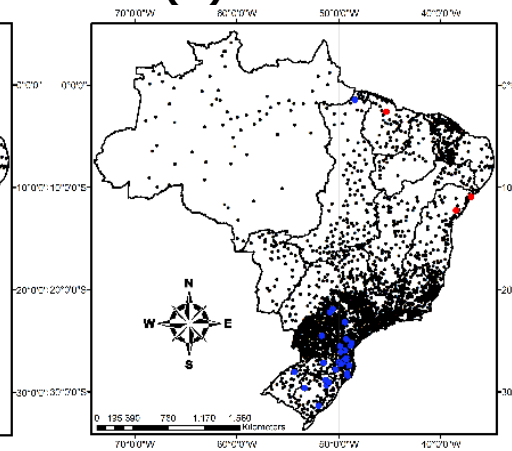

(e) FDR-MK-PW

Crescente

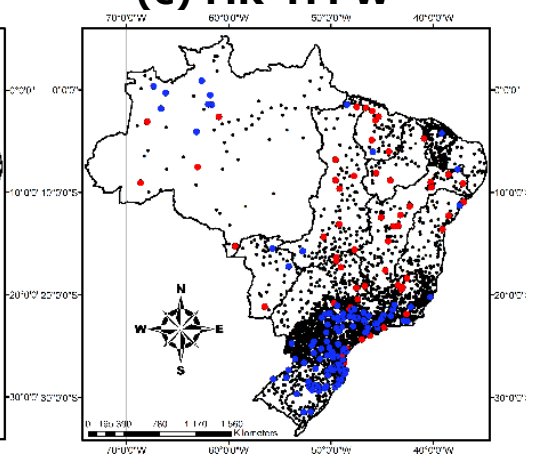

(f) FDR-MK-TFPW

Figura 4 - Distribuição espacial das estações significativas e não-significativas obtidas pelas seis diferentes estratégias de análise para o índice PRCPT. 


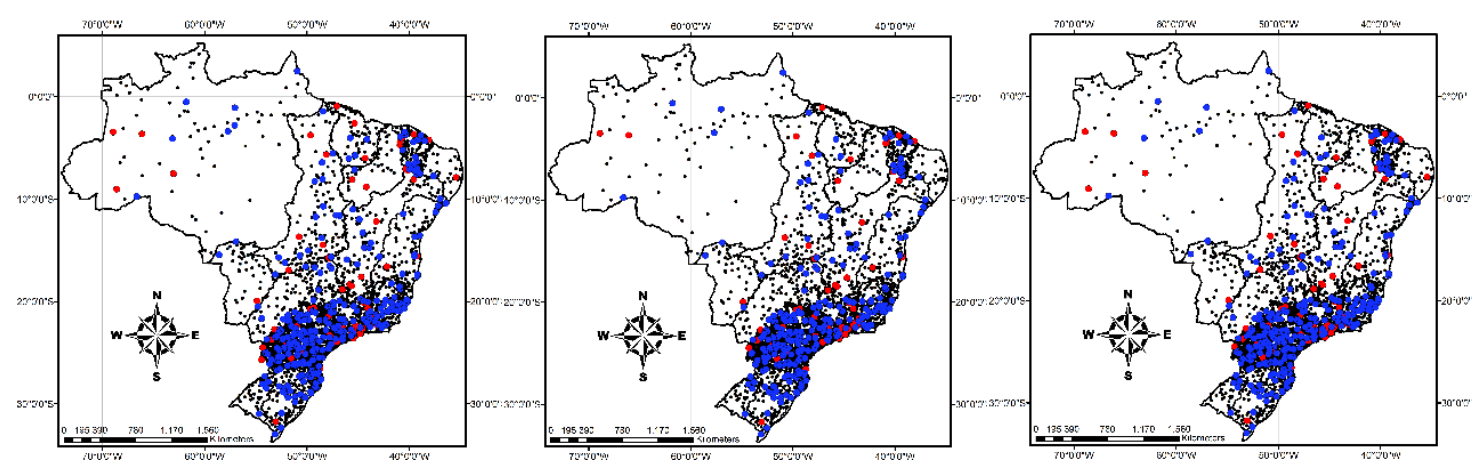

(a) MK

(b) MK-PW

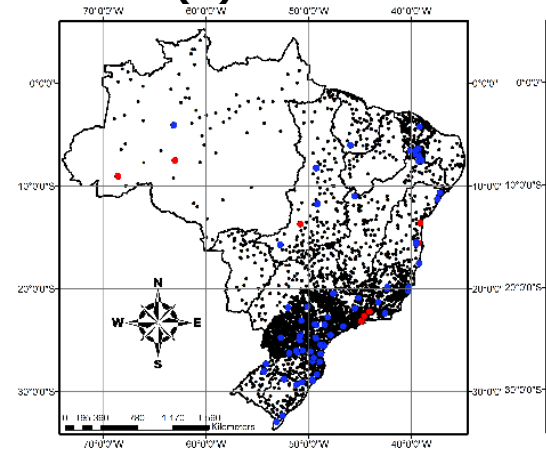

(d) FDR-MK

Crescente

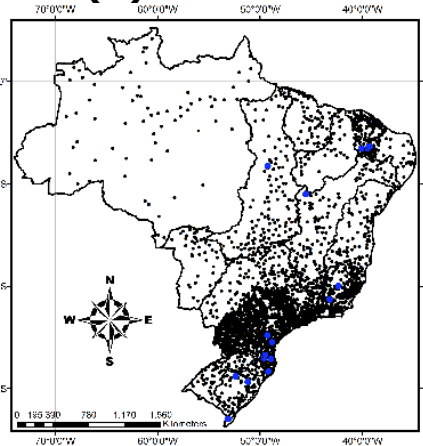

(e) FDR-MK-PW (c) MK-TFPW

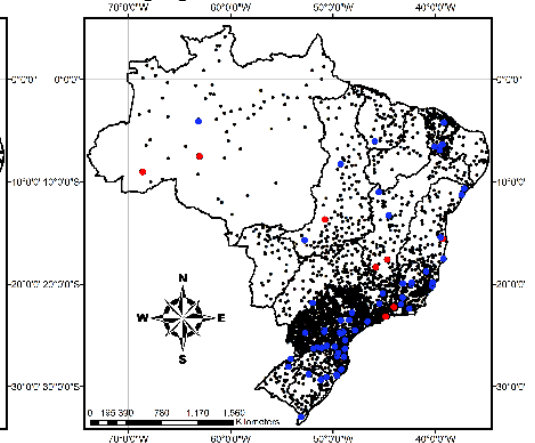

(f) FDR-MK-TFPW

Figura 5 - Distribuição espacial das estações significativas e não-significativas obtidas pelas seis diferentes estratégias de análise para o índice RX1d.

\section{CONCLUSÕES}

Os resultados do estudo de detecção de tendência de dez índices de precipitação, realizado em 2481 estações de monitoramento espalhadas em todo o território brasileiro, mostraram que tanto a correlação temporal, quanto a questão da multiplicidade, se não forem consideradas na análise de deteç̧ão, podem distorcer de forma considerável o número de estações de monitoramento identificadas com a presença de tendência, levando a uma interpretação equivocada da situação. Para a climatologia, negligenciar esses fatores resulta inexoravelmente numa superestimativa do número de estações com tendência devido ao número elevado de falsas deteç̧ões. Foi possível concluir também que a questão da multiplicidade tem um peso maior do que a da correlação temporal, o que já era esperado dado que o nível de correlação temporal em séries de índices de precipitação não costuma ser elevada. Entretanto, não é possível afirmar que a correlação temporal possa ser desprezada, visto que dependendo do método empregado para lidar com essa questão, os resultados podem ser muito díspares. Os índices utilizados, PRCPT e RX1d, tiveram impactos similares na redução dos resultados de tendência nas mais diferentes regiões analisadas, sinalizando que esse impacto independe do padrão da variabilidade pluviométrica existente.

Os resultados apresentados aqui ilustram uma parte das dificuldades existentes em estudos dessa natureza na ciência climatológica. Além da correlação temporal e da multiplicidade de testes de hipóteses, há outros fatores, não tratados neste artigo, que também afetam os resultados de um 
estudo de detecção, tais como o número reduzido de estações com séries longas e a existência de variabilidade natural de longo período, muitas vezes associadas com fenômenos climáticos de larga escala. A presença dessa última pode ser erroneamente interpretada como uma tendência monotônica, especialmente em estações com séries curtas (poucas décadas).

\section{AGRADECIMENTOS}

O presente trabalho foi realizado com o apoio da Coordenação de Aperfeiçoamento de Pessoal de Nível Superior - Brasil (CAPES) - Código de Financiamento 001, do Conselho Nacional de Desenvolvimento Científico e Tecnológico - CNPq (CHAMADA UNIVERSAL MCTI/CNPq No 01/2016), e da Fundação de Amparo à Pesquisa do Distrito Federal (FAP/DF) por meio do Edital 04/2017 de Demanda Espontânea.

\section{REFERÊNCIAS BIBLIOGRÁFICAS}

ALPERT, P. et al. The paradoxical increase of Mediterranean extrem daily rainfall in spite of decrease in total values. GEOPHYSICAL RESEARCH LETTERS, [S. I.], v. 29, p. 311-314, 2002.

ALVES, L.M. Análise estatística da sazonalidade e tendências das estações chuvosas e seca na Amazônia: Clima presente e projeções futuras. Ph.D. thesis, Instituto Nacional de Pesquisas Espaciais, São José dos Campos. 2016.

ALVES, B. C. C.; SOUZA FILHO, F. A.; SILVEIRA, C. S. Análise de Tendências e Padrões de Variação das Séries Históricas de Vazões do Operador Nacional do Sistema (ONS). RBRH - Revista Brasileira de Recursos Hídricos. Volume 18, n.4 -Out/Dez , p. 19-34. 2013.

AMORIM, Renato $S$. Detecção de Tendências em Séries de Extremos Hidrológicos Considerando Efeitos de Autocorrelação Temporal e Multiplicidade de Testes. Orientador: Dirceu Silveira Reis Júnior. 2018. 167 p. Dissertação (Mestrado em Tecnologia Ambiental e Recursos Hídricos) - Universidade de Brasília, Brasília/DF, 2018.

ÁVILA, A.; JUSTINO, F.; WILSON, A.; BROMWICH, D. e AMORIM, M. Recent precipitation trends, flash floods and landslides in southern Brazil. Environ. Res. Lett., n. 11, 114029. 2016.

BARROS, V. R.; DOYLE, M. E.; CAMILLONI, I. A. Precipitation trends in southeastern South America: relationship with ENSO phases and with low-level circulation. Theoretical and Applied Climatology. Viena, n. 93, p. 19-33, fev. 2008.

BAYAZIT, M.; ONOZ, B. To prewhiten or not prewhiten in trend analysis?. Hydrological Science Journal, [S. I.], v. 52, n. 4, p. 611-624, 2007.

BLAIN, G. C. The Mann-Kendall test: the need to consider the interaction between serial correlation and trend. Acta Scientiarum. Agronomy, [S. I.], v. 35, p. 393-402, 2013.

BLAIN, G. C. Removing the influence of the serial correlation on the MannKendall test. Revista Brasileira de Meteorologia, [S. I.], v. 29, p. 161-170, 2014. 
BENJAMINI, Y.; HOCHBERG, Y. Controlling the false Discovery rate: A practical and powerful approach to multiple testing. Journal of the Royal Statistical Society: Series B (Methodological), [S. I.], v. 57, n. 1, p. 289-300, 1995.

BOMBARDI, R. J.; CARVALHO, L. M. V. Práticas Simples em Análises Climatológicas: Uma Revisão. Revista Brasileira de Meteorologia, [S. I.], v. 32, n. 3, p. 311-320, 2017.

BUARQUE, D. C.; CLARKE, R. T.; MENDES, C. Spatial correlation in precipitation trends in the Brazilian Amazon. Journal of Geophysical Research: Atmospheres: Climate and Dynamics, [S.I.], v. 115, n. D12, 2010. DOI https://doi.org/10.1029/2009JD013329

CAMMALLERI, C.; MICALE, F.; VOGT, J. Recent temporal trend in modelled soil water deficit over Europe driven by meteorological observations. International Journal of Climatology, [S.I.], v. 36, n. 15, p. 4903-4912, 2016.

CAVALCANTI, I. F. A.; AMBRIZZI, T. Teleconexões e suas influências no Brasil. In: CAVALCANTI, I. F. A.; FERREIRA, N. J, DIAS; M. A. F., JUSTI; M. G. A. Tempo e clima no Brasil. São Paulo: Ed. Oficina de Textos, p. 317-335. 2009.

CHAGAS, V. B. P.; CHAFFE, P. L. B. The role of land cover in the propagation of rainfall into streamflow trends. Water Resources Research, [S. I.], v. 54, n. 9, p. 5986?6004, 2018.

CLARKE, R. T. On the (mis)use of statistical methods in hydro-climatological research. Hydrological Sciences Journal, [S. I.], v. 55, n. 2, 2010.

CONSELHO NACIONAL DE RECURSOS HÍDRICOS-CNRH, Instituí a Divisão Hidrográfica Nacional em regiões hidrográficas. Resolução n. 32, de 15 de outubro de 2003. Brasília, Publicado no DOU em 17/12/2003, 2003.

COX, D. R.; STUART, A. Some Quick Sign Tests for Trend in Location and Dispersion. Biometrika, [S. I.], v. 42, n. 1/2, p. 80-95, 1955.

DOUGLAS, E. M.; VOGEL, R. M.; KROLL, C. N. Trends in flood and low flows in the United States: impact of spatial correlation. Journal of Hydrology, [S. I.], v. 240, n. 1-2, p. 90-105, 2000.

ELY, D. F. Padrões Espaciais Das Tendências Das Precipitações Sazonais E Mensais No Estado Do Paraná - Brasil. Revista Brasileira de Climatologia Edição Especial - XIII Simpósio Brasileiro de Climatologia Geográfica - Curitiba, Ano 15, p. 83 - 105, Jun. 2019.

ESPINOZA, J. C.; RONCHAIL, J.; MARENGO, J. A.; e SEGURA, H. Contrasting North-South changes in Amazon wet-day and dry-day frequency and related atmospheric features (1981-2017). Clim. Dyn. n. 116, p. 1-18. 2018.

FATICHI, S.; CAPORALI, E. A comprehensive analysis of changes in precipitation regime in Tuscany. International Journal of Climatology, [S.I.], v. 29, n. 13, p. 1883-1893, 2009.

FERREIRA, P. S. et al. Avaliação Da Tendência Espaço-Temporal Da Precipitação Pluviométrica Em Uma Região Semiárida Do Estado De Pernambuco. Revista Brasileira de Climatologia. Ano 13, Vol. 21, JUL/DEZ 2017.

FERREIRA, P.S., SOUZA, W. M., SILVA, J. F. AND GOMES, V. P. Variabilidade Espaço-Temporal das Tendências de Precipitação na Mesorregião Sul Cearense e 
sua Relação com as Anomalias de TSM. Revista Brasileira de Meteorologia, v. 33 , n. 1, p. 141-152. 2018

GANGULI, P.; GANGULY, A. R. Space-time Trends in U.S. Meteorological Droughts. Journal of Hydrology: Regional Studies, [S. I.], v. 8, p. 235-259, 2016.

GUDMUNDSSON, L.; SENEVIRATNE, S. I. European Drought Trends. Proc. IAHS, [S. I.], v. 369, p. 75-79, 2015.

HAMED, K. H.; HAO, A. R. A Modified Mann-Kendall Trend Test for Autocorrelated Data. Journal of Hydrology, [S. I.], v. 294, p. 182-196, 1998.

HAMED, K. H. Exact distribution of the Mann?Kendall trend test statistic for persistent data. Journal of Hydrology, [S. I.], v. 365, p. 86-94, 2009.

HAYLOCK, M. R.; PETERSON, T. C.; ALVES, L. M.; AMBRIZZI, T.; ANUNCIAÇÃO, Y. M. T.; BAEZ, J.; BARROS, V. R.; BERLATO, M. A.; BIDEGAIN, M.; CORONEL, G.; CORRADI, V.; GARGIA, V. J.; GRIMM, A. M.; KAROLY, D.; MARENGO, J. A.; MARINO, M. B.; MONCUNILL, D. F.; NECHET, D.; QUINTANA, J.; REBELLO, E.; RUSTICUCCI, M.; SANTOS, J. L.; TREBEJO, I.; VICENT, L. A. Trends in total and extreme South American rainfall in 1960-2000 and links with sea surface temperature. Journal of Climate, Washington, v. 19, p. 1490-1512, 2006.

HIERA, M.D.; LIMA JÚNIOR, A. F.; ZANELLA, M. E. Tendência da Precipitação No Estado do Ceará. Revista Brasileira de Climatologia. Ano 15, Vol. 24, p. 300 321, JAN/JUN. 2019.

HOCHBERG, Y. A Sharper Bonferroni Procedure for Multiple Tests of Significance. Biometrika, [S. I.], v. 75, n. 4, p. 800-802, 1988.

HOLM, S. A simple sequentially rejective multiple test procedure. Scandinavian Journal of Statistics, [S. I.], v. 6, n. 2, p. 65-70, 1979.

HUMPHREY, V.; GUDMUNDSSON, L.; SENEVIRATNE, S. I. Assessing global water storage variability from GRACE: trends, seasonal cycles, subseasonal anomalies e extremes. Surveys in Geophysics, [S. I.], v. 37, n. 2, p. 357-395, 2016.

IPCC. Summary for Policymakers. Climate Change 2013: The Physical Science Basis. Contribution of Working Group to the Fifth Assessment Report of the Intergovernmental Panel on Climate Change [Stocker, T. F., D. Qin, G.-K. Plattner,M.Tignor, S.K. Allen, J. Boschung, A. Nauels, Y. Xia,V. Bexand P.M. Midgley (eds.)]. Cambridge: Cambridge University Press. 2013.

KAYANO, M.T.; CAPISTRANO, V.B. How the Atlantic multidecadal oscillation (AMO) modifies the ENSO influence on the South American rainfall. International Journal of Climatology, v. n/a, p. n/a-n/a, 2013.

KENDALL, M. G. Rank Correlation Methods. 4. ed. London: Charles Griffin, 1975.

KHALIQ, M. N.; OUARDA, T. B.; GACHON, P. Identification of temporal trends in annual and seasonal low flows occurring in Canadian rivers: The effect of shortand long-term persistence. Journal of Hydrology, [S. I.], v. 369, n. 1-2, p. 183197, 2009.

KOUTSOYIANNIS, D. Nonstationarity versus scaling in hydrology. Journal of Hydrology, [S. I.], v. 324, n. 1-4, p. 239?254, 2006. 
KULKARNI, A.; VON STORCH, H. Monte Carlo experiments on the effect of serial correlation on the Mann-Kendall test of trend. Meteorologische Zeitschrift, [S. I.], v. 4, n. 2, p. 82-85, 1995.

KUNDZEWICZ, Z. W.; ROBSON, A. J. Change detection in hydrological records?a review of the methodology. Hydrological Sciences Journal, [S. I.], v. 49, n. 1, p. 7-19, 2004.

LETTENMAIER, D. P. Detection of trends in water quality data from records with dependent observations. Water Resources Research, [S. I.], v. 12, n. 5, p. 1037-1046, 1976.

LIVEZEY, R. E.; CHEN, W. Y. Statistical Field Significance and its Determination by Monte Carlo Techniques. Monthly Weather Review, [S. I.], v. 111, p. 46-59, 1983.

MALLYA, G. et al. Trends and variability of droughts over the Indian monsoon region. Weather and Climate Extremes, [S. I.], v. 12, p. 43-68, 2016.

MANN, H. B. Nonparametric Tests Against Trend. Econometrica, [S. I.], v. 13, p. 245?259, 1945.

MARENGO, J. A.; VALVERDE, M. C. Caracterização do clima no século XX e cenário de mudanças de clima para o Brasil no século XXI usando os modelos do IPCC-AR4. Revista Multiciência, n. 9, p. 5-28. 2007.

MARENGO, J. A. et al. An intercomparison of observed and simulated extreme rainfall and temperature events during the last half of the twentieth century: Part 2: Historical trends. Climatic Change, [S. I.], v. 98, n. 3, 2010.

MARENGO, J. A., JR, S. C, THONICKE, K., BURTON, C., HALLADAY, K., BETTS, R. A., ALVES L. M. ; SOARES W. R. Changes in Climate and Land Use Over the Amazon Region: Current and Future Variability and Trends. Front. Earth Sci. 6:228. 2018

MERZ, B.; NGUYEN, V. D.; VOROGUSHYN, S. Temporal clustering of floods in Germany: Do flood-rich and flood-poor periods exist?. Journal of Hydrology, [S. I.], v. 541, p. 824-838, 2016.

MIAO, C. Y. et al. Spatio-temporal variability of streamflow in the Yellow river: possible causes and implications. Hydrological Sciences Journal, [S. I.], v. 57, n. 7, p. 1355?1367, 2012.

MÜLLER, I. I.; KRÜGER, C. M.; KAVISKI, E. Análise de estacionariedade de séries hidrológicas na bacia incremental de Itaipu. Revista Brasileira de Recursos Hídricos, [S. I.], v. 3, n. 4, p. 51-71, 1998.

ONOZ, B.; BAYAZIT, M. Block bootstrap for Mann?Kendall trend test of serially dependent data. Hydrological Processes, [S. I.], v. 26, n. 23, p. 3552-3560, 2012.

PEDRON, I. T. et al. Trends and variability in extremes of precipitation in Curitiba - Southern Brazil. International Journal of Climatology, [S. I.], v. 37, n. 3, p. $1250-1264,2017$.

PENEREIRO, J. C.; MESCHIATTI, M. C. Variabilidades e Tendências Climáticas nos Máximos do Inverno e Verão no Brasil. Revista Brasileira de Climatologia. Ano 13, v. 21, p. 378-400. JUL/DEZ 2017. 
PENEREIRO, J. C., BADINGER, A., MACCHERI, N. A. AND MESCHIATTI, M. C. Distribuições de Tendências Sazonais de Temperatura Média e Precipitação nos Biomas Brasileiros. Revista Brasileira de Meteorologia, v. 33, n. 1, p. 97-113. 2018.

PINHEIRO, A.; GRACIANO, R. L. G.; SEVERO, D. L. Tendência das series temporais de precipitação da região sul do Brasil. Revista Brasileira de Meteorologia, São José dos Campos, v. 28, n. 3, p. 281-290, 2013.

RENARD, B. et al. Regional methods for trend detection: Assessing field significance and regional consistency. Water Resources Research, [S. I.], v. 44, n. 8, p. 1-17, 2008.

ROSIN, C.; AMORIM, R. S.; MORAIS, T. S. Analysis of hydrological trends in the Rio das Mortes watershed. Brazilian Journal of Water Resources, [S. I.], v. 20, n. 4, p. 991-998, 2015.

SAlgueiro, J. H. P. B.; PINTO, E. J. A.; MONTENEGRO, S. M. G. L.; SilVA, B. B.; SOUZA, W. M.; OLIVEIRA, L. M. Influence of oceanic-atmospheric interactions on extreme events of daily rainfall in the Sub-basin 39 located in Northeastern Brazil.RBRH, Porto Alegre, v. 21, n. 4, p. 685-693, out./dez. 2016.

SALVIANO, M. F.; GROPPO, J. D.; PELLEGRINO, G. Q. Análise de tendências em dados de precipitação e temperatura no Brasil. Revista Brasileira de Meteorologia, São José dos Campos, v. 31, n. 1, p. 64-73, 2016.

SANTOS, C.A.C; BRITO, J.I.B. Analysis of indices of extremes for the semi-arid of Brazil and its relations with SST and NDVI. Rev. Bras. Meteorol., vol.22, n.3, p. 303-312, 2007.

SATYAMURTY, P; CASTRO, A. A.; TOTA, J.; GULARTE, L. E. S.; MANZI, A. O.

Rainfall trends in the Brazilian Amazon Basin in the past eight decades.

Theoretical and Applied Climatology, v. 99, n. 1-2, p. 139-148, 2010.

SERINALDI, F.; KILSBY, C. G.; LOMBARDO, F. Untenable nonstationarity: An assessment of the fitness for purpose of trend tests in hydrology. Advances in Water Resources, [S. I.], v. 111, p. 132-155, 2018.

SIMES, R. J. An improved Bonferroni procedure for multiple tests of significance. Biometrika, [S. I.], v. 73, p. 751-754, 1986.

SKANSI, M.M., BRUNET, M., SIGRÓ, J., AGUILAR, E., GROENING, J.A.A., BENTANCUR, O.J., GEIER, Y.R.C., AMAYA, R.L.C., JÁCOME, H., RAMOS, A.M., ROJAS, C.O., PASTEN, A.M., MITRO, S.S., JIMÉNEZ, C.V., MARTÍNEZ, R., ALEXANDER, L.V., JONES, P. D. Warming and wetting signals emerging from analysis of changes in climate extreme indices over South America. Global and Planetary Change, v. 100, p. 295-307, 2013.

SORIÇ, B. Statistical "Discoveries" and Effect-Size Estimation. Journal of the American Statistical Association, [S. I.], v. 84, n. 406, p. 608-610, 1989.

SUN, W. et al. False Discovery Control in Large-Scale Spatial Multiple Testing. Journal of Royal Statistical Society: Series B (Statistical Methodology), [S. I.], v. 77, n. 1, p. 59-83, 2014. 
TEIXEIRA, M.; SATYAMURTY, P. Trends in the frequency of intense precipitation events in southern and southeastern Brazil during 1960?2004. Journal of Climate, [S. I.], v. 24, n. 7, p. 1913-1921, 2011.

VALVERDE, M.C.; MARENGO, J.A. Extreme Rainfall Indices in the Hydrographic Basins of Brazil. Open Journal of Modern Hydrology, n. 4, p. 10-26. 2014.

VENTURA, V.; PACIOREK, C. J.; RISBEY, J. S. Controlling the Proportion of Falsely Rejected Hypotheses when Conducting Multiple Tests with Climatological Data. Journal of Climate, [S. I.], v. 17, n. 22, p. 4343-4356, 2004.

VON STORCH, H. Misuses of Statistical Analysis in Climate Research. In: VON STORCH, H.; NAVARRA, A. Analysis of Climate Variability. [S. I.]: Springer, 1999. cap. 2, p. 11-26. ISBN 978-3-662-03744-7. 1999.

WILKS, D. S. On "Field Significance" and the False Discovery Rate. Journal of Applied Meteorology and Climatology, [S. I.], v. 45, p. 1181-1189, 2006.

WILKS, D. S. "The Stippling Shows Statistically Significant Grid Points": How Research Results are Routinely Overstated and Overinterpreted, and What to Do about It. Bulletin of the American Meteorological Society, [S. I.], p. 2263-2273, 2016.

YUE, S. et al. The influence of autocorrelation on the ability to detect trend in hydrological series. Hydrological Processes, [S. I.], v. 16, n. 9, p. 1807-1829, 2002.

YUE, S.; WANG, C. Y. Applicability of prewhitening to eliminate the influence of serial correlation on the Mann?Kendall test. Water Resources Research, [S. I.], v. 38, n. 6, p. 4-1-4-7, 2002.

ZHANG, X. YANG, F. RClimDex (1.0) - User Manual. Climate Research Branch Environment Canada Downsview, Ontario, Canadá, 22p., 2004.

ZHANG, $X$. et al. Indices for monitoring changes in extremes based on daily temperature and precipitation data. Wires Climate Change, [S. I.], v. 2, n. 6, p. 851-870, 2011. 